\title{
Cost-Effectiveness of Pre-exposure HIV Prophylaxis During Pregnancy and Breastfeeding in Sub-Saharan Africa
}

\author{
Joan T. Price, MD, MPH, * Stephanie B. Wheeler, PhD, MPH, † Lynda Stranix-Chibanda, MBChB, MMed, $\neq$ \\ Sybil G. Hosek, PhD, \& D. Heather Watts, MD,\| George K. Siberry, MD, MPH, $\mid$ Hans M. L. Spiegel, MD,\# \\ Jeffrey S. Stringer, MD,* and Benjamin H. Chi, MD, MSc*
}

\begin{abstract}
Introduction: Antiretroviral pre-exposure prophylaxis (PrEP) for the prevention of HIV acquisition is cost-effective when delivered to those at substantial risk. Despite a high incidence of HIV infection among pregnant and breastfeeding women in sub-Saharan Africa (SSA), a theoretical increased risk of preterm birth on PrEP could outweigh the HIV prevention benefit.
\end{abstract}

Methods: We developed a decision analytic model to evaluate a strategy of daily oral PrEP during pregnancy and breastfeeding in SSA. We approached the analysis from a health care system perspective across a lifetime time horizon. Model inputs were derived from existing literature and local sources. The incremental cost-effectiveness ratio (ICER) of PrEP versus no PrEP was calculated in 2015 U.S. dollars per disability-adjusted life year (DALY) averted. We evaluated the effect of uncertainty in baseline estimates through one-way and probabilistic sensitivity analyses.

From the *Division of Global Women's Health, Department of Obstetrics and Gynecology, University of North Carolina Chapel Hill, Chapel Hill, NC; $\dagger$ Department of Health Policy and Management, University of North Carolina at Chapel Hill, Chapel Hill, NC; $\ddagger$ Department of Pediatrics and Child Health, College of Health Sciences, University of Zimbabwe, Harare, Zimbabwe; $\S$ Department of Psychiatry, John Stroger Hospital of Cook County, Chicago, IL; $\|$ Office of the Global AIDS Coordinator and Health Diplomacy, U.S. Department of State, Washington, DC; $\mid$ Eunice Kennedy Shriver National Institute of Child Health and Human Development, National Institutes of Health, Bethesda, MD; and \#Kelly Government Services, Contractor to Prevention Sciences Program, Division of AIDS, National Institute of Allergy and Infectious Diseases, National Institutes of Health, Bethesda, MD.

Investigator and trainee support for this study was provided by the Eunice Kennedy Shriver National Institute of Child Health and Human Development (NICHD; R01HD075131 and T32HD075731) and the National Institute of Allergy and Infectious Diseases (NIAID; K24AI120796). Additional support was provided by the International Maternal Pediatric Adolescent AIDS Clinical Trials Network (UM1AI068632, UM1AI068616, and UM1AI067616); funded by NIAID, NICHD, and the National Institute of Mental Health.

The conclusions and opinions expressed in this article are those of the authors and do not necessarily reflect those of the National Institutes of Health or the U.S. Department of State.

BHC received grant funding from the Gilead Foundation to support global health research training. The remaining authors have no conflicts of interest to disclose.

Supplemental digital content is available for this article. Direct URL citations appear in the printed text and are provided in the HTML and PDF versions of this article on the journal's Web site (www.jaids.com).

Correspondence to: Joan T. Price, MD, MPH, Division of Global Women's Health, University of North Carolina at Chapel Hill, CB\# 7577, Chapel Hill, NC 27599-7577 (e-mail: joan_price@med.unc.edu).

Copyright (C) 2016 Wolters Kluwer Health, Inc. All rights reserved. This is an open access article distributed under the terms of the Creative Commons Attribution-NonCommercial-NoDerivatives License 4.0 (CC BY-NC-ND), which permits downloading and sharing the work provided it is properly cited. The work cannot be changed in any way or used commercially.
Results: PrEP administered to pregnant and breastfeeding women in SSA was cost-effective. In a base case of 10,000 women, the administration of PrEP averted 381 HIV infections but resulted in 779 more preterm births. PrEP was more costly per person $(\$ 450$ versus \$117), but resulted in fewer disability-adjusted life years (DALYs) (3.15 versus 3.49). The incremental cost-effectiveness ratio of $\$ 965 /$ DALY averted was below the recommended regional threshold for cost-effectiveness of \$6462/DALY. Probabilistic sensitivity analyses demonstrated robustness of the model.

Conclusions: Providing PrEP to pregnant and breastfeeding women in SSA is likely cost-effective, although more data are needed about adherence and safety. For populations at high risk of HIV acquisition, PrEP may be considered as part of a broader combination HIV prevention strategy.

Key Words: pre-exposure prophylaxis, HIV prevention, pregnancy, breastfeeding, sub-Saharan Africa, cost-effectiveness

(J Acquir Immune Defic Syndr 2016;72:S145-S153)

\section{INTRODUCTION}

Antiretroviral (ARV) pre-exposure prophylaxis (PrEP) has been shown to prevent HIV acquisition among at-risk individuals. Daily oral tenofovir-emtricitabine (TDF-FTC) reduced up to $70 \%$ of incident HIV infections among heterosexual couples, intravenous drug users, and men who have sex with men. ${ }^{1-4}$ Although some clinical trials of PrEP use among women have shown equivocal results, these findings were likely due to variable adherence observed among study participants. ${ }^{5-7}$ With demonstrated feasibility and acceptability of PrEP in early pilot programs, ${ }^{8-10}$ the scale-up of PrEP in HIV prevention initiatives has been supported broadly by the World Health Organization (WHO), the U.S. Centers for Disease Control and Prevention, and others. ${ }^{1-13}$

Previous analyses have shown PrEP to be cost-effective when delivered to populations at highest risk of HIV infection. ${ }^{14-16}$ HIV incidence among young women in sub-Saharan Africa (SSA) is one of the highest in the world. ${ }^{17}$ Rates of HIV acquisition in this population may double during pregnancy and breastfeeding, and the risk of consequent mother-to-child transmission (MTCT) is tripled when incident maternal infection occurs during this period. ${ }^{18,19}$ Despite a clear need for primary HIV prevention in this at-risk population, there remains incomplete evidence on the safety and effectiveness of TDFFTC use by HIV-uninfected women during pregnancy and 
breastfeeding. ${ }^{11,20,21}$ Some triple-drug HIV treatment regimens have been associated with an increased risk of preterm birth (PTB) and other adverse outcomes, although the contribution of specific ARV drugs is unclear. ${ }^{22-27}$ A potential increase in PTB risk due to ARV drug exposure, coupled with low adherence and effectiveness, could negate any HIV prevention benefit of PrEP in this population. To better understand the role of HIV prophylaxis during this period of heightened risk, we conducted a cost-effectiveness analysis of oral PrEP among pregnant and breastfeeding women in SSA. An economic modeling analysis can help to identify current knowledge gaps in PrEP implementation research and inform policy decisions regionally and internationally.

\section{METHODS}

\section{Economic Model, Setting, and Population Characteristics}

We constructed a decision analytic model to compare the costs and effectiveness of the reference strategy of no PrEP against a scenario of PrEP provision to pregnant and breastfeeding women in SSA (TreeAge Pro, Williamstown, MA; Supplemental Fig. 1, http://links.lww.com/QAI/A829). We considered a lifetime time horizon and approached the analysis from the perspective of the health care system. Costs and utilities were discounted at a $3 \%$ annual rate. ${ }^{28}$ The primary outcome measure was the incremental cost-effectiveness ratio (ICER), measured as 2015 U.S. dollars (USD) per disability-adjusted life years (DALYs) averted. Cost-effectiveness was defined according to WHO guidelines as an ICER $<3$ times the per capita gross domestic product (GDP); a very cost-effective intervention requires an ICER less than the per capita GDP. ${ }^{28}$ The WHO Afro-E region of SSA (http://www.who.int/healthinfo/statistics/ gbdestimatesregionallist.xls) has an overall per capita GDP of $\$ 2154$, which translates to a cost-effectiveness threshold of \$6462/DALY averted. ${ }^{28}$

Our study population was composed of HIV-negative pregnant and breastfeeding women presenting to antenatal care (ANC). ${ }^{30,31}$ We searched the medical literature, conference abstracts, and published reports to identify setting-specific estimates for model parameters (Table 1). Where such data were unavailable or of a narrow scope, we drew estimates from our HIV care and treatment program in $\mathrm{Zambia}^{47}$ and expert opinion. Probabilities were calculated from rates using the standard equation $P=1-\mathrm{e}^{-\mathrm{rt}}$. We derived probabilities of $\mathrm{HIV}$ infection from incidence rates in pregnancy ( 4.7 per 100 personyears) and postpartum (2.9 per 100 person-years) reported by a meta-analysis of studies in SSA. ${ }^{18}$ The MTCT risk if maternal HIV infection occurs during pregnancy or breastfeeding is $22.7 \% .{ }^{18}$ For women who were infected with HIV in pregnancy but do not transmit HIV to their fetus, the MTCT risk during breastfeeding (assuming a median breastfeeding duration of 18 months) was assumed to be similar to that among women with chronic HIV, or $9 \%{ }^{43}$ The baseline PTB risk in SSA is $12 \%{ }^{45}$ HIV infection confers a risk ratio of PTB of 1.5 , which is comparable with the PTB risk in our HIV-infected Zambian cohort. $^{46,47}$ The risk of PTB among women taking PrEP is theoretical and for this analysis was inferred from a randomized

\begin{tabular}{|c|c|c|}
\hline Description & Estimate & Reference \\
\hline \multicolumn{3}{|l|}{ Baseline characteristics } \\
\hline Median age of pregnancy & 24 & 30,31 \\
\hline Life expectancy adult woman & 60 & 32 \\
\hline Life expectancy HIV+ adult woman & 48 & 31,33 \\
\hline HIV incidence in pregnancy, per 100py & 4.7 & 18 \\
\hline HIV incidence postpartum, per 100py & 2.9 & 18 \\
\hline Disability weight HIV & 0.135 & 34 \\
\hline Cost of HIV/AIDS care (includes ART), \$ per py & $\$ 1300$ & 35,36 \\
\hline \multicolumn{3}{|l|}{ Infant/child outcomes } \\
\hline Life expectancy for HIV- infant & 57 & 32 \\
\hline ART coverage HIV+ adult women & 0.43 & 17,32 \\
\hline ART coverage HIV + infants/children & 0.34 & 17,32 \\
\hline Life expectancy HIV+ infant, on ART & 37 & 37 \\
\hline $\begin{array}{l}\text { Life expectancy HIV+ infant infected peripartum, } \\
\text { no ART }\end{array}$ & 1.1 & 38 \\
\hline $\begin{array}{l}\text { Life expectancy HIV+ infant infected during BF, } \\
\text { no ART }\end{array}$ & 9.4 & 38 \\
\hline Probability neonatal death, term infant & 0.013 & 39 \\
\hline Probability neonatal death, preterm infant & 0.056 & 39 \\
\hline Mortality at $24 \mathrm{mo}$, HIV-negative exposed infant & 0.08 & $40-42$ \\
\hline Mortality at $24 \mathrm{mo}$, HIV-negative unexposed infant & 0.02 & $40-42$ \\
\hline $\begin{array}{l}\text { MTCT risk if incident infection during pregnancy/ } \\
\text { BF, } \%\end{array}$ & 22.7 & 18 \\
\hline MTCT risk during BF if infected in pregnancy, $\%$ & 9 & 18,43 \\
\hline Disability weight PTB & 0.106 & 44 \\
\hline Baseline PTB risk, \% & 12 & 45 \\
\hline PTB risk if maternal HIV infection, $\%$ & 18 & 26,46 \\
\hline PTB risk on PrEP, $\%$ & 20 & 22,47 \\
\hline Immediate cost of PTB, \$ per LBW infant & $\$ 572$ & 48,49 \\
\hline \multicolumn{3}{|l|}{ PrEP administration and monitoring } \\
\hline PrEP effectiveness & 0.55 & 50 \\
\hline Median duration in $\mathrm{ANC}$, wk & 20 & 31 \\
\hline Proportion breastfeeding & 0.96 & 51 \\
\hline Median duration breastfeeding, yrs & 1.25 & $51-53$ \\
\hline Cost of PrEP medication (TDF-FTC), \$ per py & $\$ 75$ & 54 \\
\hline $\begin{array}{l}\text { Cost of toxicity monitoring and HIV/HepB testing, } \\
\$ \text { per py }\end{array}$ & $\$ 140$ & 55 \\
\hline $\begin{array}{l}\text { Cost of adherence counseling and monitoring, } \\
\text { \$ per py }\end{array}$ & $\$ 100$ & 56 \\
\hline Discounting rate per year, $\%$ & 3.00 & 28 \\
\hline GDP per capita, WHO AfroE region & $\$ 2154 *$ & 28,29 \\
\hline
\end{tabular}

All costs are reported in 2015 USD unless otherwise noted

*Reported in 2005 International dollars.

$\mathrm{BF}$, breastfeeding; HepB, hepatitis B; ART, antiretroviral therapy; MTCT, motherto-child transmission of HIV; PTB, preterm birth; PrEP, pre-exposure HIV prophylaxis; TDF-FTC, tenofovir-emtricitabine; ANC, antenatal care; GDP, gross domestic product.

trial comparing the efficacy and safety of triple ARV regimens for the prevention of MTCT among HIV-infected women, and was also consistent with data from Zambia. ${ }^{22}$

\section{Programmatic Assumptions}

We assumed that once-daily oral PrEP medication consisting of TDF-FTC would begin at the first ANC visit with 
a negative HIV screening test and terminate with cessation of breastfeeding (median 15 months postpartum) ${ }^{51-53} \mathrm{We}$ derived the median gestational age (GA) of entry into ANC as 19 weeks and the median GA at delivery as 39 weeks from an international database. ${ }^{31} \mathrm{We}$ assumed uniform effectiveness of PrEP for each woman in the base-case analysis, based on a median time spent in ANC of 20 weeks (ie, 39-week GA at delivery minus 19-week GA at entry into ANC). Because there has been no observational study of PrEP in pregnancy, we varied widely the estimate for effectiveness of PrEP during pregnancy and breastfeeding to account for variable adherence and exposure duration. We focused on the index pregnancy only (and assumed no subsequent pregnancies occurred) and did not consider the cost or disability of subsequent transmissions beyond mother and child (eg, to sexual partners). For women who were infected with HIV during pregnancy or breastfeeding, we assumed initiation of lifelong antiretroviral therapy (ART) under recent WHO guidelines for a realistic proportion of women (43\%). ${ }^{11,17}$ Although current guidelines recommend that all infants are started on ART as soon as they are diagnosed with $\mathrm{HIV}$, true coverage of pediatric HIV treatment approximates $34 \% .{ }^{11,17,32}$ We assumed this "real world" coverage rate in our model to account for infants who fail to access timely health services and die before diagnosis or treatment.

\section{Cost Parameters}

Cost parameters were derived from international economic sources and previous cost analyses (Table 1). Where available, we used relevant purchasing power parity to convert original costs in local currency to international dollars and then inflated to 2015 USD. Given that many aspects of HIV prevention programs are paid for in USD through international funding agencies, if costs were reported in the literature only in USD with no reference to original local currency, we directly inflated these costs to 2015 USD using historical consumer price index data from the National Bureau of Labor Statistics. ${ }^{57}$

The cost of PrEP medication for the duration of pregnancy and breastfeeding was estimated from the cost of TDF-FTC negotiated by the Clinton Health Access Initiative in its list of ceiling prices. ${ }^{54}$ The cost of toxicity surveillance, based on the recommended quarterly basic metabolic panel plus HIV and hepatitis B testing, ${ }^{11}$ was micro-costed from previous economic analyses in SSA. ${ }^{15,55}$ We estimated additional PrEP program costs to include personnel and facility expenses required for adherence counseling and monitoring activities based on data from previous economic evaluations of voluntary counseling and testing strategies. ${ }^{56}$ Our composite program cost is higher than that reported by a recent analysis of PrEP for serodiscordant couples in Uganda; however, given likely broad regional variations in cost, we intentionally chose the higher estimates of programmatic costs for our model inputs. ${ }^{58}$ The annual cost of care for HIV/AIDS, including provision of ARVs, was derived from an analysis of costs of government programs in 45 sites in $\mathrm{Zambia}^{35}$ and is consistent with ranges reported in previous analyses. ${ }^{36,59,60}$ The average cost of PTB per infant was based on two studies in SSA that reported the cost of care for neonates with low birth weight as a proxy for prematurity given imprecise GA estimates. ${ }^{48,49}$ The cost of PTB was assumed to be a uniform upfront cost and was not discounted.

\section{Payoff Measures}

The primary payoff was calculated in DALY as the sum of years of life lost (YLL) and years lived with disability (YLD). We calculated YLD associated with both mother and infant outcomes using disability estimates from the Global Burden of Disease Study. ${ }^{34}$ We used the disability weight for HIV over the entire life expectancy of an HIV-positive woman or infant. Disability due to PTB was applied to the lifetime of all infants born prematurely; for preterm HIVpositive infants, we used the more severe disability weight associated with HIV to generate YLD. ${ }^{61}$

We modeled known differences in neonatal mortality between term and preterm infants. ${ }^{39}$ We accounted for differences in survival between HIV-negative infants born to infected mothers and those born to uninfected mothers by modeling differential mortality risk at 24 months of age based on HIV exposure. ${ }^{40-42}$ Data are scarce on long-term survival of HIV-infected children who initiate ART in infancy ${ }^{62}$; therefore, we modeled childhood mortality based on a previously published simulation of the effect of ART on pediatric HIV disease progression in South Africa $^{63}$ and subsequently estimated life expectancy of surviving adolescents from a Ugandan cohort. ${ }^{37}$ For HIV-positive infants not receiving treatment, we modeled life expectancies based on known survival differences between infants infected in utero or peripartum versus those infected postpartum. ${ }^{38}$

Discounting for DALY payoffs in YLL and YLD for mother and infant was performed using the following standard equations, where $\mathrm{N}$ is number of deaths, $\mathrm{r}$ is annual discount rate (eg, 3\%), I is number of incident cases, DW is disability weight, and L represents years of either standard life expectancy at age of death (in YLL equation) or duration of disability (in YLD equation). ${ }^{44}$

$$
\begin{gathered}
\mathrm{YLL}=\frac{\mathrm{N}}{0.03}\left(1-\mathrm{e}^{-0.003 \mathrm{~L}}\right) \\
\mathrm{YLD}=\frac{\mathrm{I} \times \mathrm{DW} \times\left(1-\mathrm{e}^{-0.03 \mathrm{~L}}\right)}{0.03}
\end{gathered}
$$

\section{Cost-Effectiveness and Sensitivity Analyses}

Baseline values for cost, utility, and expected survival for the PrEP scenario were used for the base-case analysis (Table 1). The ICER, our primary outcome measure, was defined as the incremental cost (in 2015 USD) per number of DALYs between a scenario of PrEP provision in pregnancy and breastfeeding versus the standard scenario of no PrEP.

We performed one-way deterministic sensitivity analyses of parameters that influenced the ICER outcome using ranges of best- and worst-case scenarios, derived from previous clinical trials, meta-analyses, or reasonable assumption. In one-way sensitivity analyses, costs were varied by a factor of at least three times the base value to account for uncertainty in 
estimates and assumed wide regional variations. Probabilities were varied according to ranges from the medical literature or to account for uncertainty in point estimates and regional variation. We performed threshold analyses to understand at which point the value of key uncertain parameters rendered PrEP not cost-effective. We determined the thresholds of PrEP effectiveness and the probability of PTB on PrEP, as the estimates for these variables were most uncertain. We conducted probabilistic sensitivity analysis (PSA) using Monte Carlo simulation to assess the confidence in our ICER outcome by varying all parameters simultaneously over distributions informed by parameter ranges reported in the literature at a minimum.

\section{RESULTS}

\section{Base Case}

In a base-case scenario of 10,000 HIV-negative pregnant women in SSA, the administration of daily oral PrEP through pregnancy and breastfeeding prevented 381 total HIV infections (485 with PrEP and 866 without PrEP); 303 maternal infections (389 with PrEP and 692 without PrEP) and 78 infant infections (96 with PrEP and 174 without PrEP) were averted. PrEP provision was associated with 779 additional PTBs (2000 with PrEP and 1221 without PrEP) than the no PrEP strategy and an associated 35 more neonatal deaths (218 with PrEP and 183 without PrEP).

\section{Incremental Costs and Utilities}

For the base-case analysis, the PrEP scenario costs an average of $\$ 333$ more per person than no PrEP ( $\$ 450$ versus $\$ 117)$. PrEP provision yielded an average 3.15 DALY compared with 3.49 DALY in the no PrEP scenario. This produced an ICER of $\$ 965$ per DALY averted in the PrEP scenario. This ICER was much lower than the threshold for cost-effectiveness of $\$ 6462$ for the region and even met the threshold of "very cost-effective," which is equal to the regional per capita GDP $(\$ 2154 / \mathrm{DALY}){ }^{28}$

\section{Sensitivity Analyses}

One-way deterministic sensitivity analyses showed that the model was most sensitive to variation in PrEP effectiveness and probability of PTB while taking PrEP (Table 2). Analyses of the remaining variables demonstrated confidence in the outcome; the PrEP strategy remained cost-effective even when varying all other variables to their highest and lowest range values.

To determine individual thresholds for cost-effectiveness of key parameters, one parameter was varied widely while all others were kept stable. Threshold analyses revealed that the threshold for PrEP effectiveness was 0.78 (ie, a risk ratio for HIV infection of 0.78 or lower among PrEP users), above which PrEP lost cost-effectiveness. The probability of PTB on PrEP at which PrEP was no longer cost-effective was 0.30.

PSAs were performed using Monte Carlo simulation by 1000 iterations; these PSA substantiated the relative robustness of the model (Fig. 1). Distributions for PSA were derived from confidence intervals presented in the literature, where available, or calculated from reasonable assumption of standard deviations. Each cost distribution was set to the range used in oneway analyses (a factor of at least 3 times the baseline input) and assigned gamma distribution. ${ }^{64} \mathrm{~A}$ gamma distribution was applied given its constraints to be positive and fully continuous. Both probability and disability distributions were set to a factor of at least 2 times the baseline input and assigned beta distributions, bounded at 0 and $1 .^{64}$ The PrEP strategy was cost-effective (with an ICER below the willingness-to-pay threshold of $\$ 6462 /$ DALY averted) in $88 \%$ of the iterations. Four percent of the simulations were above the willingness-topay threshold and $8 \%$ were dominated (in which PrEP was both more costly and less effective than no PrEP). The costeffectiveness by willingness-to-pay curve demonstrates that the PrEP strategy was very cost-effective, with an ICER of \$2154 or less, in approximately $79 \%$ of strategies (Supplemental Fig. 2, http://links.lww.com/QAI/A829).

\section{DISCUSSION}

Daily oral PrEP for the prevention of HIV among pregnant and breastfeeding women in SSA is likely costeffective. Despite upfront programmatic and drug costs, as well as a modeled elevation in PTB risk, the PrEP strategy was cost-effective relative to the no PrEP strategy due to the cost and disability of averted maternal and infant HIV infections. The ICER outcome was less than internationally accepted willingness-to-pay thresholds; moreover, the PrEP strategy met the WHO definition of very cost-effective and likely remains cost-effective even in those countries where per capita productivity is lower than in the region overall. ${ }^{28}$

Previous analyses in other high-risk groups such as men who have sex with men, female sex workers, intravenous drug users, and serodiscordant couples have shown PrEP to be cost-effective, ${ }^{15,16,65-67}$ but rarely cost-saving. ${ }^{14}$ Multiple studies on oral and topical PrEP in southern Africa have reported ICERs of less than $\$ 5000$ per QALY saved or DALY averted, with cost-effectiveness driven by adherence and prioritization of provision to those at highest risk. ${ }^{14-16,58,60}$ To date, however, cost-effectiveness of PrEP during pregnancy and breastfeeding has not been studied. Many additional factors in this population require consideration, including medication adherence during pregnancy; maternal, fetal, and infant toxicities; induced ARV drug resistance; and the potential for HIV transmission to infants (among women infected despite prophylaxis).

Our economic model was most sensitive to variation in PrEP effectiveness, which is highly dependent on medication adherence. ${ }^{50}$ In some clinical trials, low adherence among study participants has rendered PrEP ineffective altogether. ${ }^{5,6,50,68}$ Adherence to oral and vaginal regimens of PrEP has varied considerably among nonpregnant women and women with incident pregnancy., ${ }^{5,70}$ It is possible that low adherence may be attributed in part to the design of those clinical trials; most PrEP studies that reported no effect on HIV acquisition among women have been randomized, blinded placebo-controlled trials where participants were uncertain about efficacy and whether or not they received the active agent. In one open-label 
TABLE 2. One-way Deterministic Sensitivity Analyses

\begin{tabular}{|c|c|c|c|c|c|c|c|c|}
\hline Parameter & $\begin{array}{c}\text { Range } \\
\text { Minimum }\end{array}$ & $\begin{array}{l}\text { Incr } \\
\text { Cost }\end{array}$ & $\begin{array}{c}\text { DALY } \\
\text { Averted }\end{array}$ & $\begin{array}{c}\text { ICER } \\
(\$ / D A L Y)\end{array}$ & $\begin{array}{c}\text { Range } \\
\text { Maximum }\end{array}$ & $\begin{array}{l}\text { Incr } \\
\text { Cost }\end{array}$ & $\begin{array}{c}\text { DALY } \\
\text { Averted }\end{array}$ & $\begin{array}{c}\text { ICER } \\
(\$ / D A L Y)\end{array}$ \\
\hline PrEP effectiveness ${ }^{50}$ & 0.30 & $\$ 322$ & 0.66 & 485 & 0.95 & $\$ 350$ & -0.16 & Dominated \\
\hline Probability of PTB on $\operatorname{PrEP}^{22,47}$ & 0.10 & $\$ 276$ & 0.63 & 438 & 0.45 & $\$ 475$ & -0.37 & Dominated \\
\hline $\begin{array}{l}\text { Probability of HIV in } \\
\text { pregnancy }^{18}\end{array}$ & 0.01 & $\$ 342$ & 0.13 & 2646 & 0.10 & $\$ 310$ & 0.89 & 348 \\
\hline Cost of PrEP program ${ }^{55,56}$ & $\$ 80$ & $\$ 176$ & 0.35 & 509 & $\$ 720$ & $\$ 804$ & 0.35 & 2332 \\
\hline $\begin{array}{l}\text { Probability of HIV in } \\
\text { breastfeeding }\end{array}$ & 0.01 & $\$ 340$ & 0.16 & 2137 & 0.05 & $\$ 329$ & 0.45 & 732 \\
\hline Cost of $\operatorname{PrEP}^{54}$ & $\$ 25$ & $\$ 284$ & 0.35 & 823 & $\$ 225$ & $\$ 480$ & 0.35 & 1392 \\
\hline Cost of PTB ${ }^{48,49}$ & $\$ 200$ & $\$ 304$ & 0.35 & 881 & $\$ 2000$ & $\$ 444$ & 0.35 & 1287 \\
\hline
\end{tabular}

Each parameter listed was varied according to ranges noted, holding all other parameters constant. Costs and probabilities were varied widely to estimate best- and worst-case scenarios for each parameter. The order of the parameters in this table reflects the effect of each parameter on the incremental cost-effectiveness ratio (ICER) outcome- parameters at the top of the table (pre-exposure prophlyaxis (PrEP) effectiveness and probability of preterm birth (PTB) on PrEP) drove the ICER most significantly, whereas variation of parameters at the bottom affected the ICER less. Varying other parameters not listed did not affect the ICER outcome. ICERs noted to be "dominated" indicate that PrEP was both more expensive and less effective than no PrEP at that parameter value. Incr cost, incremental cost; DALY, disability-adjusted life year; ICER, incremental cost-effectiveness ratio; PrEP, pre-exposure HIV prophylaxis; PTB, preterm birth.

study among women, adherence was noted to be high (up to $76 \%$ ), particularly among those on once-daily oral dosing. ${ }^{10}$ Open-label implementation studies offering PrEP to pregnant and breastfeeding women could optimize PrEP effectiveness, at relatively low cost per participant, by adherence monitoring and counseling on the effectiveness of PrEP if taken consistently. Finally, prioritization of PrEP initiation early in pregnancy would maximize prophylactic exposure and PrEP

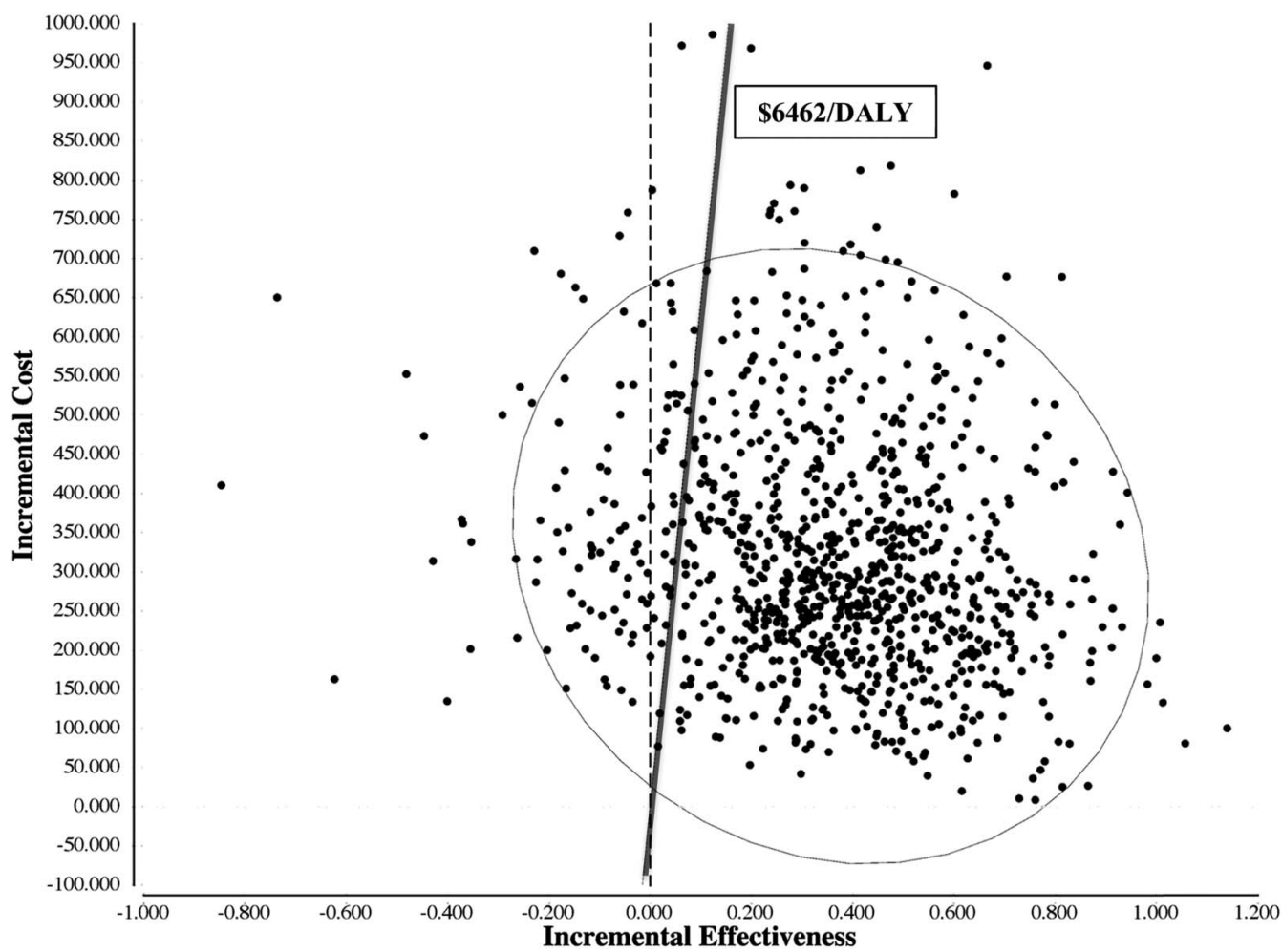

FIGURE 1. Probabilistic sensitivity analysis of incremental cost-effectiveness of pre-exposure prophylaxis (PrEP) vs. no PrEP, shown in a scatter plot. Each point estimate represents one iteration (total 1000) of incremental cost and incremental effectiveness of the PrEP strategy versus no PrEP, based on the range of parameter distributions. The oval represents the $95 \%$ confidence interval of all 1000 iterations. The solid line represents the willingness-to-pay threshold of $\$ 6462$ per DALY, or 3 times the regional per capita GDP. Any point to the left of the solid line represents an incremental cost-effectiveness ratio (ICER) above the willingness-to-pay threshold. Approximately $88 \%$ of all estimated ICER iterations demonstrated cost-effectiveness of the PrEP strategy. No points fell below the horizontal axis and therefore no iterations demonstrated cost savings. Points to the left of the dashed vertical axis (8\% of all iterations) indicate increased cost and less effectiveness of the PrEP strategy (ie, dominance of PrEP by no PrEP). 
effectiveness. Data from future observational studies with participants enrolled at different GAs of pregnancy will allow us to determine GA thresholds at which initiating PrEP is not cost-effective.

Our analysis suggests that the benefits of maternal and neonatal HIV prevention from PrEP outweigh even a substantially increased risk of PTB from ARV drug exposure. This increase in PTB among women taking PrEP is theoretical. To date, there has been no increase in adverse pregnancy outcomes observed among pregnant women on TDF for hepatitis B infection. ${ }^{71}$ Similarly, PrEP has been shown to be safe among women who became pregnant while taking PrEP, although the study drug was discontinued immediately when pregnancy was diagnosed (at a median GA of 5 weeks). ${ }^{20}$ An increased risk of adverse pregnancy outcomes has been found among HIV-infected women on certain triple-drug treatment regimens, ${ }^{23,24,26}$ particularly those containing protease inhibitors. ${ }^{25,72}$ Results from the PROMISE trial demonstrated a risk of PTB of up to $20 \%$ among HIV-positive pregnant women taking combination ARV regimens to prevent MTCT, which is consistent with analyses from our own data in Zambia. ${ }^{22,47}$ Despite our conservative assumption that PrEP users in our cohort would experience this same elevated risk of PTB, administration of PrEP in pregnancy remained cost-effective. The threshold PTB risk at which PrEP lost cost-effectiveness was quite high at $30 \%$. If demonstrated in clinical studies, however, this risk could preclude PrEP from being a viable prevention option in pregnancy.

The background risk of HIV infection in pregnancy and breastfeeding drove the ICER only modestly across its range values (Table 2), suggesting that PrEP may be cost-effective in areas of even lower HIV incidence. While WHO guidelines recommend PrEP for populations with HIV incidence of 3 per 100 person-years or higher, the incidence threshold at which PrEP becomes cost-effective is influenced by the PrEP-ART cost ratio and PrEP effectiveness. ${ }^{11,13,50}$ Furthermore, this incidence threshold is likely lowered in scenarios where PrEP can reduce the risk of HIV acquisition in more than one individual (eg, in the mother-infant pair). However, because the cost-effectiveness of PrEP is closely linked to the broader HIV epidemic context, we anticipate that as expanding ART coverage and other prevention efforts reduce HIV incidence in SSA, modification of this analysis will be necessary.

Among HIV-infected pregnant and breastfeeding women, the risks of combination ARV regimens on maternal and fetal outcomes are outweighed by significant benefits to both mother and child. These risks have not been thoroughly evaluated in the context of TDF-FTC for HIV prevention among uninfected pregnant and breastfeeding women. The use of PrEP has been associated with mild and transient decreases in renal function that resolve after PrEP discontinuation. ${ }^{5,73,74}$ Renal toxicity of PrEP regimens may be more severe in pregnancy, however, during which a physiologic increase in GFR balances changes in systemic and renal hemodynamics characterized by marked volume expansion and vasodilation. Additionally, fetal tenofovir exposure in utero and through breast milk may be associated with decreased infant bone mineral content and growth; but longitudinal data are sparse. ${ }^{21,75-79}$ Early results from IMPAACT P1084s, a substudy of PROMISE, showed that use of combination ARV drug regimens during pregnancy resulted in lower infant bone mineral content at birth compared with antenatal zidovudine and intrapartum nevirapine. When tenofovirbased ART was compared with other combination regimens in subset analyses, however, no differences were observed. ${ }^{80}$ Given uncertainty regarding maternal and infant toxicity of PrEP in pregnancy and breastfeeding - and the evident safety in nonpregnant populations - we did not consider additional costs or disability from these potential complications. As new data emerge from PrEP implementation studies, such assumptions may need to be revisited and incorporated into future cost-effectiveness models.

In studies among PrEP users with incident HIV infection, ARV drug resistance has been rare and has occurred primarily when PrEP was initiated in a patient with unrecognized HIV infection. ${ }^{81}$ In a WHO meta-analysis, the overall risk of resistance in PrEP studies was reported as $0.1 \%$, much lower than the background transmitted resistance of nearly $3 \%$ in SSA. ${ }^{11,82}$ Mathematical modeling has predicted that the cumulative risk of resistance due to PrEP would be lower than the risk of resistance associated with treating the HIV infections that would otherwise occur. ${ }^{83}$ For these reasons, we did not estimate additional costs or disability attributable to ARV drug resistance acquired in the setting of HIV infection despite prophylaxis. Health care providers should emphasize HIV testing before initiation of PrEP. Surveillance for resistant infection is also needed for women and their infants who do become infected with HIV despite PrEP usage. As new data emerge in this critical area, particularly from programmatic settings, our analyses may need to be refined accordingly.

This model has several limitations. First, because no clinical studies have evaluated the use of PrEP in pregnancy and breastfeeding, the PrEP effectiveness model parameter was derived from a pooled analysis of multiple short-term studies among diverse populations. ${ }^{11,13}$ "Real world" PrEP effectiveness can vary substantially based on medication efficacy, adherence, and timing of initiation, which in turn drive the cost-effectiveness of program implementation. Second, we used the disability weight of HIV only and did not account for additional disability due to AIDS, a conservative approach that could underestimate the effectiveness of PrEP. Although the universality of disability weights from the Global Burden of Disease study has been debated, ${ }^{34,84-86}$ these weights are nevertheless recommended by the WHO for analyses in resource-limited settings. ${ }^{28}$ Third, we derived a uniform cost of PTB from two small regional studies that, while consistent, may not be generalizable to the entire SSA region. The cost of PTB may differ substantially with advances in resources and capacity for neonatal intensive care. ${ }^{48,49}$ Furthermore, the cost of PTB certainly varies according to GA at delivery, but this was held constant in our analysis. A more nuanced model would consider the variance of cost and disability based on prematurity severity. To accomplish this, however, better data are required concerning GA distributions and true PTB risk in the context of PrEP use. Fourth, we assumed lifelong ART for only a proportion of HIV-infected adult women and infants, based on current global coverage estimates. Although our 
model outcome was not significantly affected by variation of individual ART coverage assumptions, expanded ART coverage across SSA could reduce the cost-effectiveness of various prevention strategies including PrEP. Fifth, we did not account for costs or disabilities of subsequent HIV infections beyond those described in our current model. Finally, we estimated costs for drugs, monitoring, and adherence support that in reality could vary significantly across countries and regions and depend on external donor funding.

Despite optimistic results from this initial economic analysis, additional considerations of participant demand, health education, local affordability, donor funding, and social equity preferences are crucial components of decision-making when resources are scarce. ${ }^{87-89} \mathrm{In}$ this analysis, we considered PrEP as a single intervention; however, there is increasing evidence that combination strategies hold greatest promise for primary HIV prevention. ${ }^{90}$ The scale-up of other HIV prevention strategies in pregnancy, for both women and their partners, may be comparable or even superior alternatives to the addition of PrEP alone. ${ }^{14,16,55,58,60}$ In settings with finite resources, the opportunity costs of any single prevention strategy require careful consideration. While this initial model shows cost-effectiveness over no PrEP, further analysis should evaluate how PrEP for pregnant and breastfeeding women may be included in the optimal mix of a multistrategy prevention program in SSA. ${ }^{89,91}$

In summary, our analysis suggests that provision of PrEP for HIV prevention among pregnant and breastfeeding women in SSA may be cost-effective, even in scenarios of high PTB risk and modest PrEP effectiveness. Concerns regarding medication adherence, viral resistance, maternal and fetal toxicity, and PTB require empirical clarification through large-scale implementation studies. Based on the existing evidence - and from a perspective of resource allocationPrEP provision to pregnant and breastfeeding women at high risk of infection could be a promising addition to combination HIV prevention programs.

\section{REFERENCES}

1. Baeten JM, Donnell D, Ndase P, et al. Antiretroviral prophylaxis for HIV prevention in heterosexual men and women. $N$ Engl J Med. 2012;367: 399-410.

2. Thigpen MC, Kebaabetswe PM, Paxton LA, et al. Antiretroviral preexposure prophylaxis for heterosexual HIV transmission in Botswana. $N$ Engl J Med. 2012;367:423-434.

3. Choopanya K, Martin M, Suntharasamai P, et al. Antiretroviral prophylaxis for HIV infection in injecting drug users in Bangkok, Thailand (the Bangkok Tenofovir Study): a randomised, double-blind, placebocontrolled phase 3 trial. Lancet. 2013;381:2083-2090.

4. Grant RM, Lama JR, Anderson PL, et al. Preexposure chemoprophylaxis for HIV prevention in men who have sex with men. N Engl J Med. 2010; 363:2587-2599.

5. Marrazzo JM, Ramjee G, Richardson BA, et al. Tenofovir-based preexposure prophylaxis for HIV infection among African women. $N$ Engl J Med. 2015;372:509-518.

6. Van Damme L, Corneli A, Ahmed K, et al. Preexposure prophylaxis for HIV infection among African women. N Engl J Med. 2012;367:411-422.

7. Rees H, Delany-Moretlwe SA, Lombard C, et al. FACTS 001 Phase III Trial of Pericoital Tenofovir 1\% Gel for HIV Prevention in Women. Seattle, WA: CROI; 2015.

8. Molina JM, Capitant C, Spire B, et al. On-demand preexposure prophylaxis in men at high risk for HIV-1 infection. $N$ Engl J Med. 2015;373:2237-2246.
9. McCormack S, Dunn DT, Desai M, et al. Pre-exposure prophylaxis to prevent the acquisition of HIV-1 infection (PROUD): effectiveness results from the pilot phase of a pragmatic open-label randomised trial. Lancet. 2016;387:53-60.

10. Bekker LG, Grant R, Hughes J, et al. HPTN 067/ADAPT Cape Town: a comparison of daily and nondaily PrEP dosing in African women. Conference on Retroviruses and Opportunistic Infections, Seattle, WA, 23-26 February 2015.

11. World Health Organization. Consolidated Guidelines on the Use of Antiretroviral Drugs for Treating and Preventing HIV Infection: Recommendations for a Public Health Approach. 2015. Available at: http://www.who.int/hiv/pub/guidelines/arv2013/download/en/index. html. Accessed January 25, 2016.

12. Centers for Disease Control and Prevention. Pre-exposure Prophylaxis for the Prevention of HIV Infection in the United States. 2014. Available at: http://www.cdc.gov/hiv/pdf/prepguidelines2014.pdf. Accessed January 26, 2016.

13. UNICEF. Consultation on Clinical, Ethical and Operational Considerations for the Implementation of Oral Pre-exposure Prophylaxis (PrEP) in Sexually Active Older Adolescents (15-19) at High Risk of HIV Infection: Meeting Report. 2015. Available at: http://www.emtct-iatt.org/wp-content/uploads/ 2015/09/PrEP-Use-in-Adolescents-Consultation-Report-Vancouver-CanadaJuly-2015.pdf. Accessed December 7, 2015.

14. Alistar SS, Grant PM, Bendavid E. Comparative effectiveness and costeffectiveness of antiretroviral therapy and pre-exposure prophylaxis for HIV prevention in South Africa. BMC Med. 2014;12:46.

15. Walensky RP, Park JE, Wood R, et al. The cost-effectiveness of preexposure prophylaxis for HIV infection in South African women. Clin Infect Dis. 2012;54:1504-1513.

16. Hallett TB, Baeten JM, Heffron R, et al. Optimal uses of antiretrovirals for prevention in HIV-1 serodiscordant heterosexual couples in South Africa: a modelling study. Plos Med. 2011;8:e1001123.

17. Joint United Nations Programme on HIV/AIDS. The Gap Report. 2014. Available at: http://www.unaids.org/sites/default/files/media_asset/UNAIDS_ Gap_report_en.pdf. Accessed January 26, 2016.

18. Drake AL, Wagner A, Richardson B, et al. Incident HIV during pregnancy and postpartum and risk of mother-to-child HIV transmission: a systematic review and meta-analysis. Plos Med. 2014;11: e1001608.

19. Mugo NR, Heffron R, Donnell D, et al. Increased risk of HIV-1 transmission in pregnancy: a prospective study among African HIV-1serodiscordant couples. AIDS. 2011;25:1887-1895.

20. Mugo NR, Hong T, Celum C, et al. Pregnancy incidence and outcomes among women receiving preexposure prophylaxis for HIV prevention: a randomized clinical trial. JAMA. 2014;312:362-371.

21. Ehrhardt S, Xie C, Guo N, et al. Breastfeeding while taking lamivudine or tenofovir disoproxil fumarate: a review of the evidence. Clin Infect Dis. 2015;60:275-278

22. Fowler MG, Qin M, Fiscus SA, et al. Efficacy and safety of 2 strategies to prevent perinatal HIV transmission. Conference on Retroviruses and Opportunistic Infections, Seattle, WA, 23-24 February, 2015.

23. Short CE, Douglas M, Smith JH, et al. Preterm delivery risk in women initiating antiretroviral therapy to prevent HIV mother-to-child transmission. HIV Med. 2014;15:233-238.

24. Darak S, Darak T, Kulkarni S, et al. Effect of highly active antiretroviral treatment (HAART) during pregnancy on pregnancy outcomes: experiences from a PMTCT program in western India. AIDS Patient Care STDS. 2013;27:163-170.

25. Watts DH, Williams PL, Kacanek D, et al. Combination antiretroviral use and preterm birth. J Infect Dis. 2013;207:612-621.

26. Chen JY, Ribaudo HJ, Souda S, et al. Highly active antiretroviral therapy and adverse birth outcomes among HIV-infected women in Botswana. J Infect Dis. 2012;206:1695-1705.

27. Townsend C, Schulte J, Thorne C, et al. Antiretroviral therapy and preterm delivery-a pooled analysis of data from the United States and Europe. BJOG. 2010;117:1399-1410.

28. Tan-Torres Edejer T, Baltussen R, Adam T, et al. Making Choices in Health: WHO Guide to Cost-effectiveness Analysis. Geneva, Switzerland: World Health Organization; 2003.

29. The World Factbook. 2013. Available at: https://www.cia.gov/library/ publications/the-world-factbook/rankorder/2004rank.html. Accessed January 26, 2016. 
30. Moodley D, Esterhuizen T, Reddy L, et al. Incident HIV infection in pregnant and lactating women and its effect on mother-to-child transmission in South Africa. J Infect Dis. 2011;203:1231-1234.

31. Demographic and Health Surveys DHS. STATcompiler Database. Available at: http://beta.statcompiler.com. Accessed November 23, 2015.

32. World Health Organization. Global Health Observatory Data Repository. Available at: www.who.int/gho/en/. Accessed December 23, 2015.

33. Eligibility for ART in lower income countries collaboration, Wandel S, Egger M, et al. Duration from seroconversion to eligibility for antiretroviral therapy and from ART eligibility to death in adult HIV-infected patients from low and middle-income countries: collaborative analysis of prospective studies. Sex Transm Infect. 2008;84(suppl 1):i31-i36.

34. Salomon JA, Vos T, Hogan DR, et al. Common values in assessing health outcomes from disease and injury: disability weights measurement study for the Global Burden of Disease Study 2010. Lancet. 2012;380: 2129-2143.

35. Marseille E, Giganti MJ, Mwango A, et al. Taking ART to scale: determinants of the cost and cost-effectiveness of antiretroviral therapy in 45 clinical sites in Zambia. PLoS One. 2012;7:e51993.

36. Bertozzi S, Gutierrez JP, Opuni M, et al. Estimating resource needs for HIV/AIDS health care services in low-income and middle-income countries. Health Policy. 2004;69:189-200.

37. Mills EJ, Bakanda C, Birungi J, et al. Life expectancy of persons receiving combination antiretroviral therapy in low-income countries: a cohort analysis from Uganda. Ann Intern Med. 2011;155:209-216.

38. Marston M, Becquet R, Zaba B, et al. Net survival of perinatally and postnatally HIV-infected children: a pooled analysis of individual data from sub-Saharan Africa. Int J Epidemiol. 2011;40:385-396.

39. Marchant T, Willey B, Katz J, et al. Neonatal mortality risk associated with preterm birth in East Africa, adjusted by weight for gestational age: individual participant level meta-analysis. PLoS Med. 2012;9:e1001292.

40. Marinda E, Humphrey JH, Iliff PJ, et al. Child mortality according to maternal and infant HIV status in Zimbabwe. Pediatr Infect Dis J. 2007; 26:519-526.

41. Shapiro RL, Lockman S, Kim S, et al. Infant morbidity, mortality, and breast milk immunologic profiles among breast-feeding HIV-infected and HIV-uninfected women in Botswana. J Infect Dis. 2007;196:562-569.

42. Newell ML, Coovadia H, Cortina-Borja M, et al. Mortality of infected and uninfected infants born to HIV-infected mothers in Africa: a pooled analysis. Lancet. 2004;364:1236-1243.

43. The Breastfeeding and H. I. V. International Transmission Study Group, Coutsoudis A, Dabis F, et al. Late postnatal transmission of HIV-1 in breast-fed children: an individual patient data meta-analysis. J Infect Dis. 2004;189:2154-2166.

44. Murray CJ, Lopez A. The Global Burden of Disease. A Comprehensive Assessment of Mortality and Disability from Diseases, Injuries, and Risk Factors in 1990 and Projected to 2020. Boston, MA: The Harvard School of Public Health; 1996.

45. Blencowe H, Cousens S, Oestergaard MZ, et al. National, regional, and worldwide estimates of preterm birth rates in the year 2010 with time trends since 1990 for selected countries: a systematic analysis and implications. Lancet. 2012;379:2162-2172.

46. Wedi CO, Kirtley S, Hopewell S, et al. Perinatal outcomes associated with maternal HIV infection: a systematic review and meta-analysis. Lancet HIV. 2015;3:e33-e48.

47. Chi BH, Vwalika B, Killam WP, et al. Implementation of the Zambia electronic perinatal record system for comprehensive prenatal and delivery care. Int J Gynaecol Obstet. 2011;113:131-136.

48. Tongo OO, Orimadegun AE, Ajayi SO, et al. The economic burden of preterm/very low birth weight care in Nigeria. $J$ Trop Pediatr. 2009;55: 262-264.

49. Sicuri E, Bardaji A, Sigauque B, et al. Costs associated with low birth weight in a rural area of Southern Mozambique. PLoS One. 2011;6:e28744.

50. Fonner VA, Dalglish SL, Kennedy CE, et al. Effectiveness and safety of oral HIV pre-exposure prophylaxis (PrEP) for all populations: A systematic review and meta-analysis. AIDS. 2016. [epub ahead of print].

51. Lauer JA, Betran AP, Victora CG, et al. Breastfeeding patterns and exposure to suboptimal breastfeeding among children in developing countries: review and analysis of nationally representative surveys. BMC Med. 2004;2:26.

52. Tomasoni LR, Galli M, Declich S, et al. Knowledge, attitudes and practice (KAP) regarding newborn feeding modalities in HIV-infected and HIV-uninfected pregnant women in sub-Saharan Africa: a multicentre study. Int Health. 2011;3:56-65.

53. Bradley SEK, Mishra V. HIV and Nutrition Among Women in SubSaharan Africa. DHS Analytical Studies No. 16. Calverton, MD: Macro International Inc; 2008.

54. Clinton Health Access Initiative (CHAI). Antiretroviral Ceiling Price List. 2014. Available at: http://45.55.138.94/content/uploads/2015/05/ CHAI-ARV-Ceiling-Price-2014-Final_English.pdf. Accessed December 23, 2015.

55. Cremin I, Alsallaq R, Dybul M, et al. The new role of antiretrovirals in combination HIV prevention: a mathematical modelling analysis. AIDS. 2013;27:447-458.

56. Sweat M, Gregorich S, Sangiwa G, et al. Cost-effectiveness of voluntary HIV-1 counselling and testing in reducing sexual transmission of HIV-1 in Kenya and Tanzania. Lancet. 2000;356:113-121.

57. Bureau of Labor Statistics. Consumer Price Index. Available at: http:// www.bls.gov/cpi/. Accessed January 25, 2016.

58. Ying R, Sharma M, Heffron R, et al. Cost-effectiveness of preexposure prophylaxis targeted to high-risk serodiscordant couples as a bridge to sustained ART use in Kampala, Uganda. J Int AIDS Soc. 2015;18(4 suppl 3):20013.

59. Menzies NA, Berruti AA, Berzon R, et al. The cost of providing comprehensive HIV treatment in PEPFAR-supported programs. AIDS. 2011;25:1753-1760.

60. Pretorius C, Stover J, Bollinger L, et al. Evaluating the cost-effectiveness of pre-exposure prophylaxis (PrEP) and its impact on HIV-1 transmission in South Africa. PLoS One. 2010;5:13646.

61. World Health Organization. WHO Methods and Data Sources for Global Burden of Disease Estimates 2000-2011. Department of Health Statistics and Information Systems. Geneva, Switzerland. 2013. Available at: http:// www.who.int/healthinfo/statistics/GlobalDALYmethods_2000_2011. pdf. Accessed December 23, 2015.

62. Collins IJ, Jourdain G, Hansudewechakul R, et al. Long-term survival of HIV-infected children receiving antiretroviral therapy in Thailand: a 5-year observational cohort study. Clin Infect Dis. 2010;51:1449-1457.

63. Johnson LF, Davies MA, Moultrie H, et al. The effect of early initiation of antiretroviral treatment in infants on pediatric AIDS mortality in South Africa: a model-based analysis. Pediatr Infect Dis J. 2012;31:474-480.

64. Gold MR, Siegel JE, Russel LB, et al. Cost-effectiveness in Health and Medicine. New York, NY: Oxford University Press; 1996.

65. Alistar SS, Owens DK, Brandeau ML. Effectiveness and cost effectiveness of oral pre-exposure prophylaxis in a portfolio of prevention programs for injection drug users in mixed HIV epidemics. PLoS One. 2014;9:e86584.

66. Juusola JL, Brandeau ML, Owens DK, et al. The cost-effectiveness of preexposure prophylaxis for HIV prevention in the United States in men who have sex with men. Ann Intern Med. 2012;156:541-550.

67. Nichols BE, Boucher CA, van Dijk JH, et al. Cost-effectiveness of preexposure prophylaxis (PrEP) in preventing HIV-1 infections in rural Zambia: a modeling study. PLoS One. 2013;8:e59549.

68. Gandhi M, Glidden DV, Liu A, et al. Strong correlation between concentrations of tenofovir (TFV) emtricitabine (FTC) in hair and TFV diphosphate and FTC triphosphate in dried Blood spots in the PrEx open label extension: implications for pre-exposure prophylaxis adherence monitoring. J Infect Dis. 2015;212:1402-1406.

69. Matthews LT, Heffron R, Mugo NR, et al. High medication adherence during periconception periods among HIV-1-uninfected women participating in a clinical trial of antiretroviral pre-exposure prophylaxis. J Acquir Immune Defic Syndr. 2014;67:91-97.

70. Matthews LT, Sibeko S, Mansoor LE, et al. Women with pregnancies had lower adherence to $1 \%$ tenofovir vaginal gel as HIV preexposure prophylaxis in CAPRISA 004, a phase IIB randomized-controlled trial. PLoS One. 2013;8:e56400.

71. Greenup AJ, Tan PK, Nguyen V, et al. Efficacy and safety of tenofovir disoproxil fumarate in pregnancy to prevent perinatal transmission of hepatitis B virus. $J$ Hepatol. 2014;61:502-507.

72. Townsend CL, Cortina-Borja M, Peckham CS, et al. Antiretroviral therapy and premature delivery in diagnosed HIV-infected women in the United Kingdom and Ireland. AIDS. 2007;21:1019-1026.

73. Mugwanya KK, Wyatt C, Celum C, et al. Changes in glomerular kidney function among HIV-1-uninfected men and women receiving emtricitabine-tenofovir disoproxil fumarate preexposure prophylaxis: a randomized clinical trial. JAMA Intern Med. 2015;175:246-254. 
74. Solomon MM, Lama JR, Glidden DV, et al. Changes in renal function associated with oral emtricitabine/tenofovir disoproxil fumarate use for HIV pre-exposure prophylaxis. AIDS. 2014;28:851-859.

75. Ransom CE, Huo Y, Patel K, et al. Infant growth outcomes after maternal tenofovir disoproxil fumarate use during pregnancy. J Acquir Immune Defic Syndr. 2013;64:374-381.

76. Siberry GK, Jacobson DL, Kalkwarf HJ, et al. Lower newborn bone mineral content associated with maternal use of tenofovir disoproxil fumarate during pregnancy. Clin Infect Dis. 2015;61:996-1003.

77. Siberry GK, Williams PL, Mendez H, et al. Safety of tenofovir use during pregnancy: early growth outcomes in HIV-exposed uninfected infants. AIDS. 2012;26:1151-1159.

78. Gibb DM, Kizito H, Russell EC, et al. Pregnancy and infant outcomes among HIV-infected women taking long-term ART with and without tenofovir in the DART trial. PLoS Med. 2012;9:e1001217.

79. Benaboud S, Pruvost A, Coffie PA, et al. Concentrations of tenofovir and emtricitabine in breast milk of HIV-1-infected women in Abidjan, Côte d'Ivoire, in the ANRS 12109 TEmAA study, Step 2. Antimicrob Agents Chemother. 2011;55:1315-1317.

80. Siberry GK, Tierney C, Stranix-Chibanda L, et al. Impact of maternal tenofovir use on HIV-exposed newborn bone mineral. Conference on Retroviruses and Opportunistic Infections, Boston, MA, 22-25 February, 2016.

81. Grant RM, Liegler T. Weighing the risk of drug resistance with the benefits of HIV preexposure prophylaxis. J Infect Dis. 2015;211:1202-1204.

82. Rhee SY, Blanco JL, Jordan MR, et al. Geographic and temporal trends in the molecular epidemiology and genetic mechanisms of transmitted
HIV-1 drug resistance: an individual-patient- and sequence-level metaanalysis. PLoS Med. 2015;12:e1001810.

83. Supervie V, Garcia-Lerma JG, Heneine W, et al. HIV, transmitted drug resistance, and the paradox of preexposure prophylaxis. Proc Natl Acad Sci U S A. 2010;107:12381-12386.

84. James KC, Foster SD. Weighing up disability. Lancet. 1999;354:87-88.

85. Jelsma J, Chivaura VG, Mhundwa K, et al. The global burden of disease disability weights. Lancet. 2000;355:2079-2080.

86. Ustun TB, Rehm J, Chatterji S, et al. Multiple-informant ranking of the disabling effects of different health conditions in 14 countries. WHO/ NIH Joint Project CAR Study Group. Lancet. 1999;354:111-115.

87. Johri M, Norheim OF. Can cost-effectiveness analysis integrate concerns for equity? Systematic review. Int J Technol Assess Health Care. 2012; 28:125-132.

88. Rutstein SE, Price JT, Rosenberg NE, et al. Hidden costs: The ethics of cost-effectiveness analyses for health interventions in resource-limited settings. Glob Public Health. 2016. [epub ahead of print].

89. Gomez GB, Borquez A, Case KK, et al. The cost and impact of scaling up pre-exposure prophylaxis for HIV prevention: a systematic review of cost-effectiveness modelling studies. PLoS Med. 2013;10: e1001401.

90. Brown G, Reeders D, Dowsett GW, et al. Investigating combination HIV prevention: isolated interventions or complex system. J Int AIDS Soc. 2015; 18:20499.

91. Baltussen RM, Hutubessy RC, Evans DB, et al. Uncertainty in costeffectiveness analysis. Probabilistic uncertainty analysis and stochastic league tables. Int J Technol Assess Health Care. 2002;18:112-119. 